\title{
Ox-LDL-Induced MicroRNA-155 Promotes Autophagy in Human Endothelial Cells via Repressing the Rheb/ mTOR Pathway
}

\author{
Jinlin Lv $^{\mathrm{a}}$ Lixia Yang ${ }^{\mathrm{b}}$ Ruiwei Guo ${ }^{\mathrm{b}}$ Yankun Shib ${ }^{\text {Ziwei Zhang }}{ }^{\mathrm{a}}$ Jinshan Ye ${ }^{\mathrm{b}}$ \\ aDepartment of Postgraduate, Kunming Medical University, Kunming, Yunnan, ${ }^{\mathrm{b} D e p a r t m e n t}$ of \\ Cardiology, Kunming General Hospital of Chengdu Military Area, Yunnan, China
}

\section{Key Words}

Microrna-155 • Autophagy $•$ Endothelial cells $・$ Rheb $\bullet$ mTOR $•$ Atherosclerosis

\begin{abstract}
Background/Aims: Autophagy, an evolutionary conserved biological process, is activated in cells to cope with various types of stress. MicroRNAs control several activities related to autophagy. However, the role of autophagy-related microRNAs during atherosclerosis is far from known. MicroRNA-155 was identified to be a crucial regulator of atherosclerosis. The objectives of the study were to analyze the effect of microRNA-155 on autophagic signaling and explore its mechanism in human endothelial cells under ox-LDL stress. Methods: The study included human endothelial cells surrogate EA.hy926 lines (EA.hy926 cells). The expression of microRNA-155 was analyzed by quantitative reverse transcription polymerase chain reaction (qRT-PCR). The effect of microRNA-155 on endothelial autophagy was observed along with the expression levels of Rheb, LC3B, Beclin1, and P62/SQSTM1 by western blotting (WB) and immunofluorescence through microRNA-155 overexpression or inhibition. Bioinformatics analysis and Luciferase reporter assay were used to explore the target gene of microRNA-155. Cell viability and apoptosis were examined by 3-[4,5-dimethylthiazol-2-yl]-5- [3-carboxymethoxyphenyl]-2-[4-sulfophenyl]-2H-tetrazolium inner salt (MTS) assay and TdT-mediated dUTP Nick-End Labeling (TUNEL) apoptosis assay. Results: MicroRNA-155 expression was significantly increased under ox-LDL stress. MicroRNA-155 increased autophagic activity, while inhibition of it alleviated ox-LDL-induced autophagy in EA.hy926 endothelial cells. In addition, dual-luciferase reporter assays showed that microRNA-155 suppressed Rheb transcription. MicroRNA-155 increased autophagic activity in EA.hy926 cells via inhibition of Rheb-mediated mTOR/P70S6kinase/4EBP signaling pathway. Furthermore, we demonstrated that microRNA-155 could regulate not only autophagy but also apoptosis in EA.hy926 cells. Conclusions: MicroRNA-155 works as a regulator of endothelial function under ox-LDL stress, making it a potential candidate for the novel therapeutic strategies against atherosclerotic diseases.




\section{Introduction}

Atherosclerosis continues to be the leading cause of morbidity and mortality worldwide [1]. The key cellular mechanisms underlying atherosclerosis include hyperlipidemia, induced inflammation, phenotypic changes in smooth-muscle cells (SMCs) and endothelial dysfunction [2]. Accumulating evidence suggests that the endothelial dysfunction caused by vascular injury appears to be an causative initiating event in atherosclerosis [3]. Meanwhile, it was found that the endothelial function regulators are vital in mediating autophagy in atherosclerosis.

Autophagy is an evolutionary conserved cellular catabolic process that delivers cytoplasmic materials to the lysosome for degradation in eukaryotic cells [4]. It plays an important role in the degradation of damaged organelles and extra biological molecules for reconstruction of the cellular structure [5]. Successful autophagy assists, in the progression of cytogenesis, in cell survival and protects cell from dysfunction [6]. Nevertheless, highly activated autophagy conversely destroys major proportions of cell organelles, and may even lead to autophagic cell death (type II programmed cell death) [7, 8]. Recent studies uncovered the underlying mechanism of autophagy, triggered by oxidized lipids, pro-inflammation factors, and metabolic stress conditions in controlling diverse cardiovascular functions [9, 10]. Similar to the process of apoptosis, autophagy is also a multistage progression that is subjected to gene regulation under some physiologic or pathologic circumstances [11].

MicroRNAs (miRNAs, MIRs) are a family of short non-coding RNA molecules, which modulate gene expression by post-transcriptional regulation, and result in either translation inhibition or RNA degradation [12]. These small molecules are generally expressed abundantly in normal tissues, and are often dysregulated in several diseases $[13,14]$. MicroRNA-155 (MIR-155) is a typical multi-functional microRNA, which is highly expressed in atherosclerotic lesions [15]. It was reported that microRNA-155 takes part in the formation of foam cell by targeting HBP1 and Bcl6, which consecutively enhance inflammatory response in atherosclerosis. The underlying mechanism involves the activation of NF- $\mathrm{KB}$ signaling via targeting SOCS1, which in turn increases tumor necrosis factor- $\alpha$ (TNF- $\alpha$ ) translation, and decreases endothelial nitric oxide synthase (eNOS) expression in endothelial cells [16-19]. Nevertheless, contradictory results exist with regard to the protective nature of microRNA-155 in atherosclerosis. It was demonstrated that microRNA-155 acts as an antiinflammatory factor by repressing calcium-regulated heat stable protein- 1 and NF- $\kappa B$ p 65 to suppress endothelial injury $[20,21]$. Anyhow, these previous findings coincide with our results which identified that microRNA-155 is significantly up-regulated following exposure of endothelial cells to ox-LDL, and correlates with atherosclerosis.

The regulatory role of microRNAs in cellular autophagy has been validated since 2009 [22]. In recent years, microRNA-155 has been paid more attention in autophagic processes. For example, Wang et al. illustrated that microRNA-155 enhances autophagy-mediated mycobacterial elimination in macrophages [23]. Chen et al. reported that microRNA-155 activates autophagy in osteosarcoma cells, and then ameliorates the anticancer drug-induced cell proliferation [24]. Moreover, several studies have showed that microRNA (miRNA)-based therapeutics could limit off-target effects and emerge as novel therapeutic agents in various human diseases $[25,26]$. However, the effect of microRNA-155 on autophagy in endothelial cells and its mechanism still remains unknown. Hence, in the present study, we investigated the role of microRNA-155 in autophagy regulation in EA.hy926 endothelial cells to find out new remedy for atherosclerosis and other vascular diseases.

\section{Materials and Methods}

Cell culture and treatment

Human endothelial cells surrogate EA.hy926 lines (EA.hy926 cells) were obtained from the Cell Bank of the Chinese Academy of Sciences (Shanghai, China). Cells were cultured as routine in Dulbecco's 


\section{Cellular Physiology Cell Physiol Biochem 2017;43:1436-1448 \\ \begin{tabular}{l|l|l} 
and Biochemistry $10.1159 / 000481875$ & $\begin{array}{l}\text { C) } 2017 \text { The Author(s). Published by S. Karger AG, Basel } \\
\text { www.karger.com/cpb }\end{array}$ \\
\hline
\end{tabular}}

Lv et al.: MicroRNA-155-Rheb and Endothelial Autophagy

Modified Eagle's high glucose medium (DMEM) (Biological Industries, 01-054-1A) supplemented with $10 \%$ inactivated fetal bovine serum (Biological Industries, 03-031-1B) and 10U/ml penicillin-streptomycin (Biological Industries, 04-001-1A) in a $5 \% \mathrm{CO}_{2}$-humidified incubator at $37^{\circ} \mathrm{C}$. Cells at $60 \%$ confluence were activated by oxidized-LDL (ox-LDL), (Yiyuanbiotech Ltd, YB- 002).

\section{Target prediction}

The conventional online programs, including Targetscan (http://www. targetscan.org), PicTar (http:// pictar.mdc-berlin.de/) and miRanda (http: //www. microrna.org/microrna/ home. do) were used to predict the targets of microRNA-155. The targets predicted by these programs were further analyzed and demonstrated by the biological experiments.

\section{RNA transfection}

All synthetic microRNAs, small interference RNA (siRNA), and their respective negative control (NC) were purchased from Shanghai GenePharma Co. Ltd. EA.hy926 cells were transiently transfected with lipofectamine $^{\mathrm{TM}} 2000$ (Invitrogen, 11668-019) according to the manufacturer's protocol. FAM-labeled cells could be observed using an Olympus BX51 fluorescence microscope (Tokyo, Japan). The transfected RNA oligonucleotide sequences are as follows: has-microRNA-155 mimics, sense 5'-UUAAUGCUAAUCGUGAUAG GGGU-3', anti-sense 5'-CCCUAUCACGAUUAGCAUUA AUU-3'; mimics-NC, sense 5'-UUCUCCGAACGUGUCACGU TT-3', antisense 5'-ACGUGCACGUUCGGA GAATT-3'; has-microRNA-155 inhibitor, 5'-ACCCCUAUCACGAUUAG CAUUAA-3'; inhibitor-NC, 5'-CAGUACUUUUGUGUAGUACAA-3'; siRNA- Rheb, sense 5'-GAAAGGGUGAUCAGU UAUGTT-3', antisense 5'-CAUAACUGAUCACCCUUUCTT- 3'; siRNA-NC, sense 5'-UUCUCCGAACGUGUCACGUT T-3', antisense 5'-ACGUGACACGUUCGGA GAATT-3'.

\section{Quantitative reverse transcription polymerase chain reaction ( $q R T-P C R$ )}

Total RNA was isolated using Eastep ${ }^{\mathrm{TM}}$ total RNA extraction kit (Promage, Shanghai LS1030). We performed qRT-PCR analyses for mRNA using TransScript green one-step qRT-PCR kit (Transgen biotech, AQ211-01). GAPDH was used as an internal reference control. We performed qRT-PCR analyses for microRNA-155 using Hairpin-it ${ }^{\mathrm{TM}}$ microRNA and U6 snRNA normalization RT-PCR quantitation kit (GenePharma, E22002) in an ABI 7500 fast system (applied biosystems CA, USA). In short, $2 \mu \mathrm{g}$ total RNAs were reverse transcribed to cDNA, then qPCR was performed using specific primer set to examine expression of mRNAs and microRNA-155. The qRT-PCR results were calculated using the comparative threshold cycle $(\mathrm{Ct})$ method. Specific primers used in the present study are as follows: has-microRNA-155, sense 5'-TAATGCTAATCGTGATAGGG-3', antisense 5'-TTTGGCACTAGCAC ATT-3'; U6 snRNA, sense 5'-CTCGCT TCGGCAGCACA-3', antisense 5'-AACGCTTCACGAATTTG CGT-3'; mTOR, sense 5'-AGGCCGCATTGTCTCTATCAA -3', antisense 5'-GCAGTAAATGCAGGTAGTCATCCA-3'; GAPDH: sense 5'-CCAGAACATCATCCCTGC-3', antisense 5'-GGAAGGCCATGCC AGTGAGC-3'.

\section{Construction of luciferase plasmids and reporter assay}

The 3'untranslated region (UTR) of Rheb fragment containing putative binding sites for microRNA-155 was amplified by PCR from human genomic DNA and cloned into pRL-TK reporter vector (Promega, E2241). The predicted target site was mutated by site-directed mutagenesis. For luciferase reporter assays, the wildtype (WT) or mutated (MUT) luciferase plasmids and microRNAs were co-transfected into cells and each experiment was repeated in triplicate. Cells were lysed at 30h post-transfection and luciferase activities were assayed by a dual-Luciferase Reporter System (Promega, E1960). Firefly luciferase activity was normalized to Renilla luciferase activity, with ratios of firefly luciferase vlaues/renilla.

\section{Western blot}

Cells were lysed in ice-cold radio immunoprecipitation assay (RIPA) lysis buffer (Byotime Ltd, P0013B), supplemented with Phenylmethanesul-fonyl (PMSF) (Byotime Ltd, ST-506). Total protein concentration of cell lysate was detected using BCA protein assay (Byotime Ltd, P0010) at 562nm on a spectrophotometer (Thermo Scientific). Cell extracts were resolved by sodium dodecyl sulfate-polyacrylamide gel electropheresis (SDS-PAGE). Antibodies used for western blotting were as follows: MAP1LC3B (1:1000 Proteintech, 187251-AP), Rheb (1:1000 Proteintech, 5924-1-AP), mTOR (1:1000 Proteintech, 20657-1-AP), Beclin-1 (1:2000 Proteintech, 11306-1-AP), P62 (1:1000 Proteintech, 18725-1-AP), phospho-mTOR (Ser2431) (1:1000 cell 


\section{Cellular Physiology Cell Physiol Biochem 2017;43:1436-1448 \begin{tabular}{l|l|l} 
DOI: 10.1159/000481875 & $\begin{array}{l}\text { O 2017 The Author(s). Published by S. Karger AG, Basel } \\
\text { www.karger.com/cpb }\end{array}$
\end{tabular}}

Lv et al.: MicroRNA-155-Rheb and Endothelial Autophagy

signaling technology, \#2974), Phospho-p70S6Kinase (Ser371) (1:1000 cell signaling technology, \#9208), Phospho-4EBP1 (Thr37/46) (1:800 cell signaling technology, \#2855), Phospho-S6 (Ser235/236) (1:1000 cell signaling technology, \#2211). Following incubation with horseradish peroxidase (HRP)-goat anti-rabbit IgG (1:4000 Proteintech, SA00001-2). An antibody against ACTB ( $\beta$-actin) (1:4000 Proteintech, 20536-1AP) served as an endogenous reference. We detected the immunofluorescence signal using Immobilon $^{\text {TM }}$ Western chemiluminescent ECL kit (Millipore WBKLS 0100) and protein ratios were calculated following Image J densitometric analysis.

\section{Cell staining for immunofluorescence microscopy}

Transfected EA.hy926 cells were fixed with 4\% paraformaldehyde for 15 min and blocked with 3\% goat serum for $20 \mathrm{~min}$ at room temperature, then incubated with Beclin-1 antibody (1:100 Proteintech, 18725-1-AP) for overnight at $4^{\circ} \mathrm{C}$. The next day, cells were washed in $0.1 \mathrm{~mol} / \mathrm{L}$ phosphate buffered saline (PBS) for 3 times and then incubated with fluorescein-conjugated affinipure goat anti-rabbit IgG (1:200 Proteintech, SA00003-2) for 1 hour at $37^{\circ} \mathrm{C}$ and subsequently stained the nuclei with DAPI. Finally, cells were observed using an Olympus BX51 fluorescence microscope (Tokyo, Japan).

\section{Cell viability assay}

Cell viability was evaluated by MTS (3-[4, 5-dimethylthiazol-2-yl]-5-[3-carboxy-methoxyphenyl]-2-[4sulfophenyl]-2H-tetrazolium, inner salt) analysis according to the manufacturer's instructions. Briefly, transfected cells were incubated in 96-well plates in a final volume of $200 \mathrm{ul}$ at the concentration of $1 \times 10^{4}$ / well for $12 \mathrm{~h}$. Then the medium was discarded, and cells were washed twice with PBS. Fresh medium containing 10\% MTS (Promega, G3582) reaction solution was added into each well, and spectrophotometric absorbance was determined after $4 \mathrm{~h}$ at OD490.

\section{Flow cytometry analysis}

Apoptosis was examined using the TUNEL (TdT-mediated dUTP Nick- End Labeling) apoptosis detection kit (Roche, Mannheim, Germany) to monitor the DNA fragmentation and analyzed by a FAC Scanflow cytometer (BD, USA) in accordance with the manufacturer's instructions.

\section{Statistical analyses}

Statistical analyses were performed using SPSS 17.0 software (SPSS Inc.). The significance of differences observed in samples was determined using Student's 2-tailed t-test or one-way ANOVA. All quantitative results were shown as means \pm standard deviation (SD) of at least 3 independent experiments. Values of $\mathrm{P}<$ 0.05 were considered to be statistically significant.

\section{Results}

\section{Ox-LDL induces microRNA-155 up-regulation in endothelial cells}

Ox-LDL is a pivotal atherogenic inducer in endothelial activation [27]. To investigate the function of microRNA-155 in endothelial autophagy, we initially detected the expression of microRNA-155 in EA.hy926 endothelial cells under ox-LDL stress. Cells were stimulated by ox-LDL at various concentrations $(0,10,20,40$ and $80 \mu \mathrm{g} / \mathrm{ml})$ for 24 hours or at different times $(0,6,12,24$, and $48 \mathrm{~h})$ at a concentration of $80 \mu \mathrm{g} / \mathrm{ml}$. As shown, ox-LDL stimulation induced significant microRNA-155 up-regulation in time and dose-dependent manner (Fig. $\left.1 \mathrm{~A}-\mathrm{B},{ }^{* *} \mathrm{P}<0.01\right)$.

\section{MicroRNA-155 induces autophagy in endothelial cells}

To regulate microRNA-155 expression in EA.hy926 endothelial cells, we transfected microRNA-155 mimics or inhibitor into EA.hy926 endothelial cells for 24h, and qRT-PCR was applied to evaluate the transfection efficiency. The results revealed that microRNA-155 expression was significantly higher in microRNA-155 mimic group and lower in microRNA-155 inhibitor group compared with their respective negative controls (NC) and blank groups (Fig. 2A-B, ${ }^{* * *} \mathrm{P}<0.001$ ). But no significant differences were observed between 


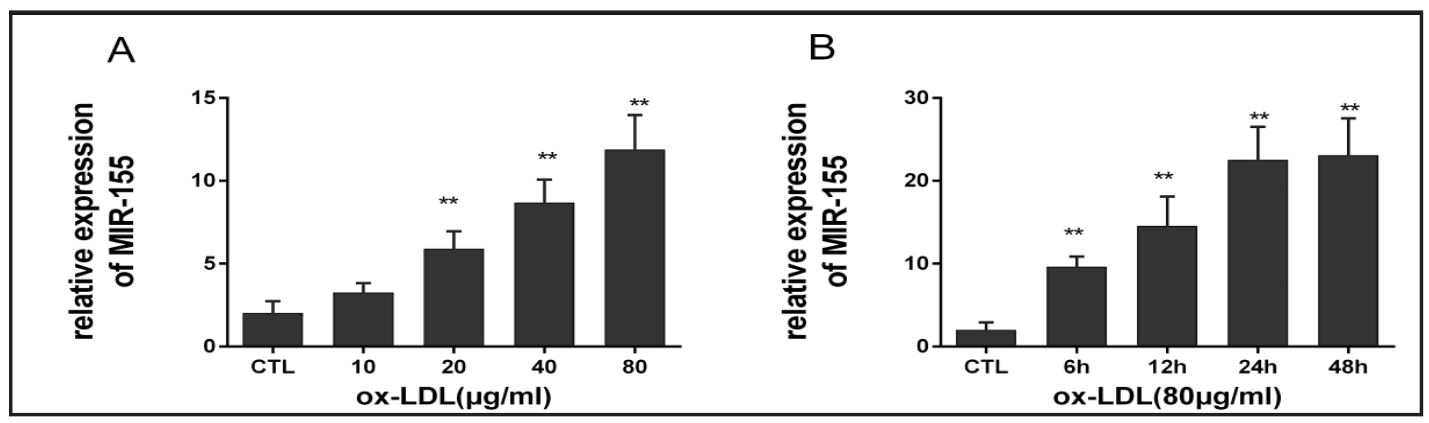

Fig. 1. Ox-LDL induces MiR-155 up-regulation. EA.hy926 cells were exposed to ox-LDL at various concentrations (A) or different times(B). Cells were collected for qRT-PCR to quantify the expression of MiR- 155. ${ }^{* *} \mathrm{P}<0.01$ versus the medium control group (CTL).

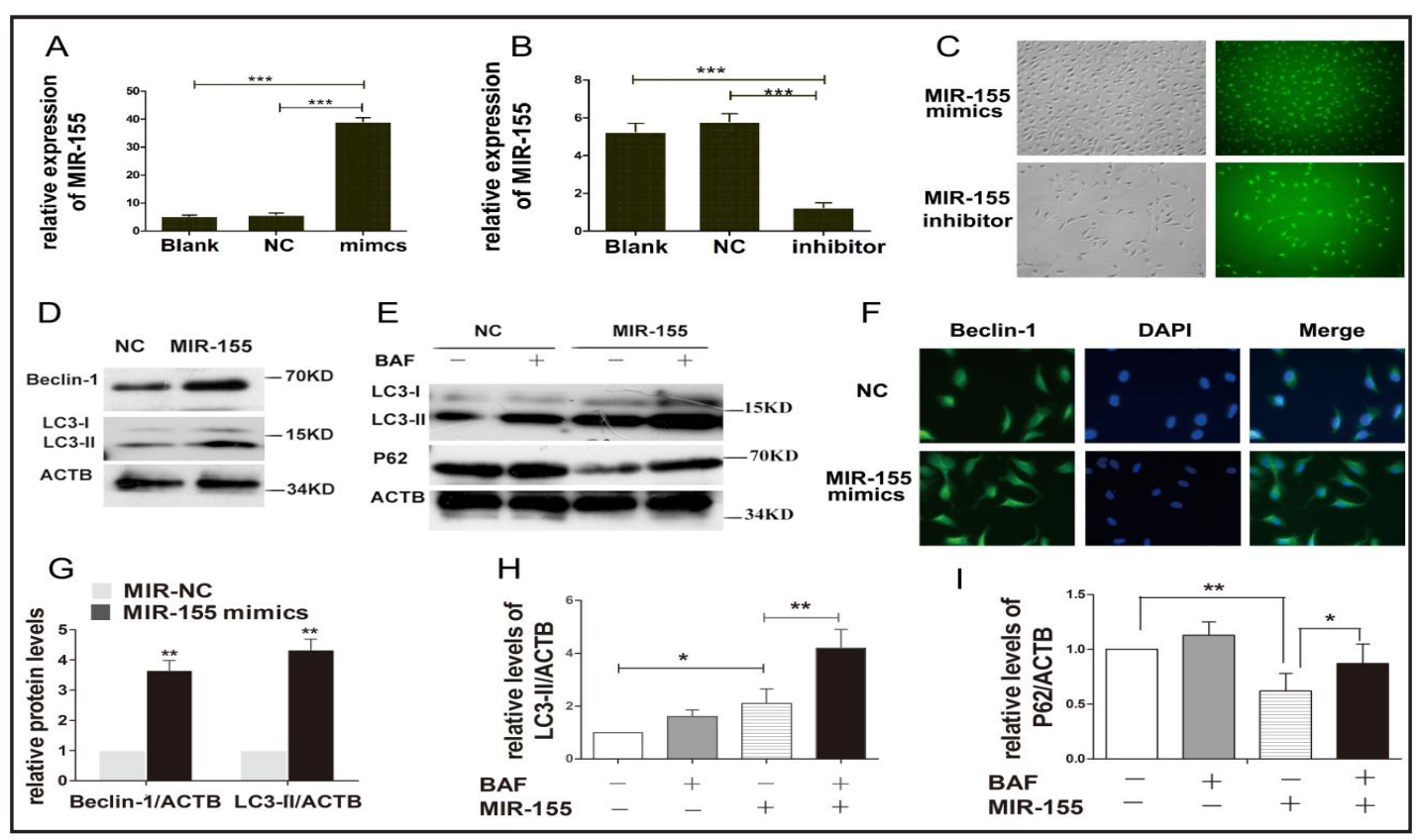

Fig. 2. MiR-155 induces autophagy in EA.hy926 endothelial cells. (A-B) MiR-155 expression was higher in MiR-155 mimics group and lower in MiR-155 inhibitor group ( $\left.{ }^{* * *} \mathrm{P}<0.001\right)$. (C) The FAM fluorescence labeling indicated more than $90 \%$ of cells treated with either the mimics or inhibitor oligos were transfected. (D and G) Transfected cells were collected for WB to detect LC3 conversion and Beclin-1 protein levels. Protein ratios were calculated following Image J densitometric analysis, $\left({ }^{* *} \mathrm{P}<0.01\right)$. (F) Immuno-staining for Beclin-1. Proteins were strongly positive in MIR-155 mimics-transfected cells. Nuclei stained with DAPI appeared in blue. (E, H and I) Transfected cells were cultured with BAF for $1 \mathrm{~h}$ at $23 \mathrm{~h}$ post transfection. Cells were collected for WB to detect LC3-II and P62 protein levels. Protein ratios were calculated following Image J densitometric analysis, $\left({ }^{*} \mathrm{P}<0.05,{ }^{* *} \mathrm{P}<0.01\right)$.

negative control and blank groups. Besides, FAM fluorescence labeling indicated that more than $90 \%$ of cells treated with either the mimics or inhibitor oligos were transfected. (Fig. 2C).

To explore the role of microRNA-155 in autophagy, we detected microtubule associated protein 1 light chain 3-I/light chain 3-II (LC3I/LC3II) and Beclin-1 levels by western blot (WB), which have been long recognized as two crucial autophagic vacuole markers [28]. Results demonstrated that microRNA-155 led to a significant increase of LC3-II and Beclin-1 levels (Fig. 2D and G, ${ }^{* *} \mathrm{P}<0.01$ ). To further demonstrate the results, we observed the effects 


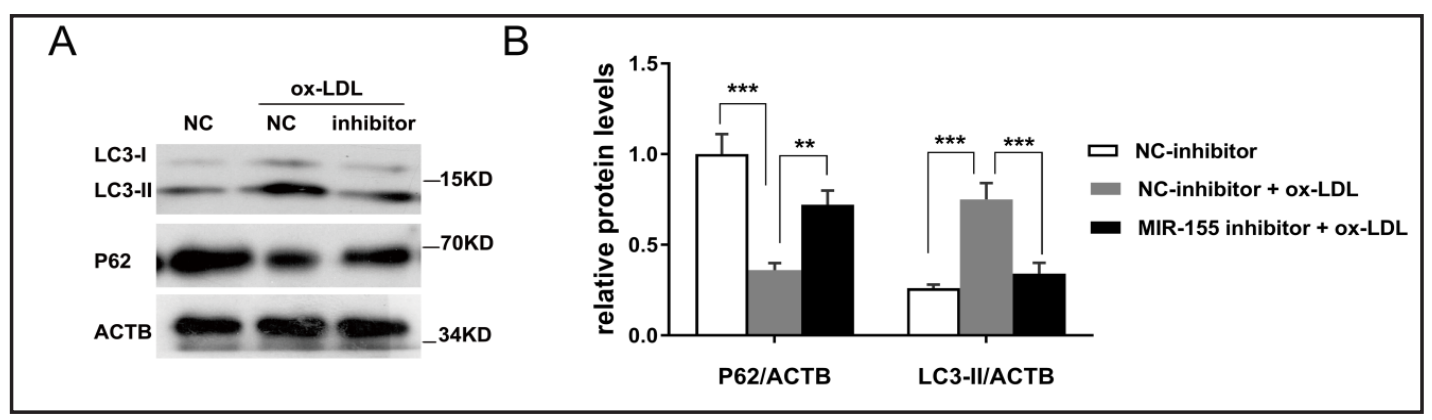

Fig. 3. Inhibition of MiR-155 suppresses ox-LDL-induced autophagy in EA.hy926 cells. (A) Transfected cells were cultured with ox-LDL (80ug/ml) for $24 \mathrm{~h}$, and then harvested for WB. (B) Ox-LDL stimulation increased LC3-II and decreased P62 levels, but inhibition of MiR-155 could reverse it. Protein ratios were calculated following Image J densitometric analysis. ( ${ }^{* *} \mathrm{P}<0.01$, $\left.{ }^{* *} \mathrm{P}<0.001\right)$.

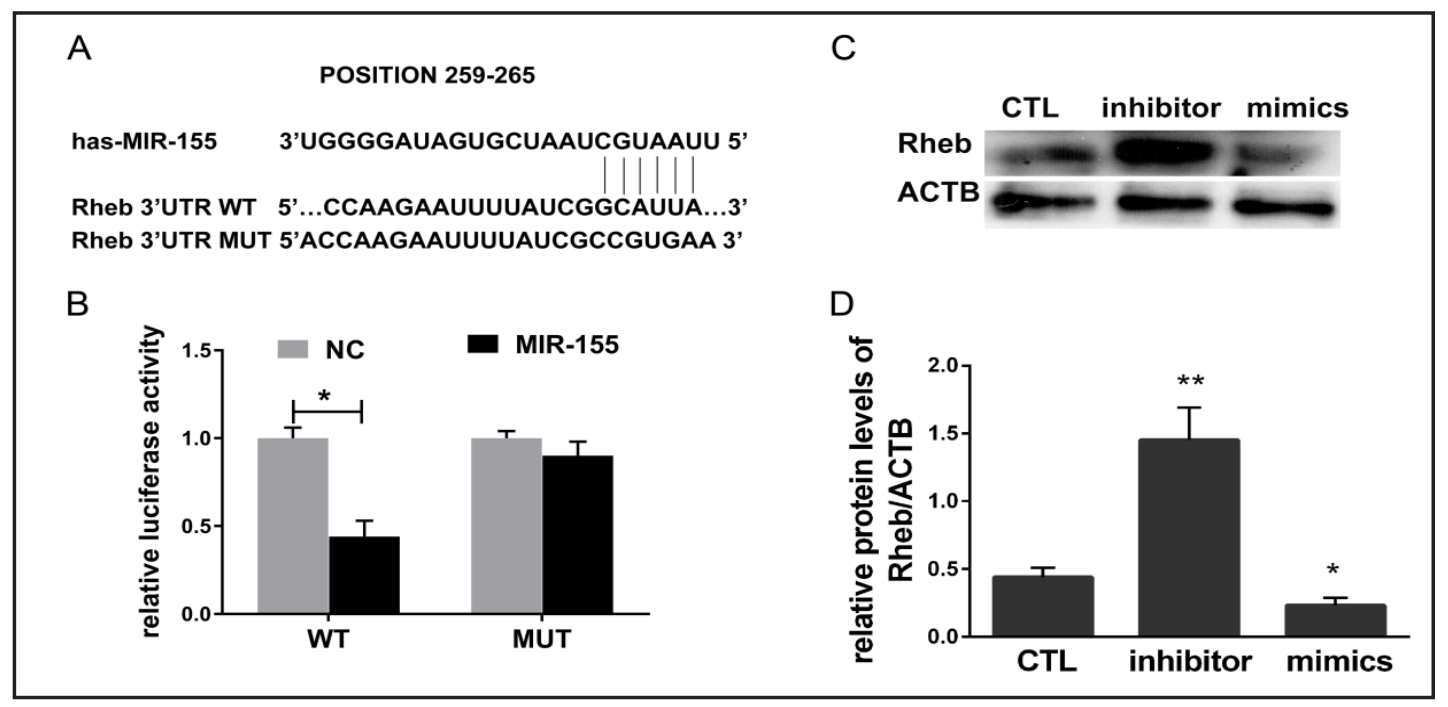

Fig. 4. MiR-155 directly interacts with the 3'-UTR of Rheb. (A) Predicted binding sequences between miR155 and seed matches in Rheb-3'UTR. (B) The luciferase activity ratios were presented as firefly luciferase vlaues/renilla luciferase values, $\left({ }^{*} \mathrm{P}<0.05\right)$. (C-D) Effects of miR-155 mimic and inhibitor on Rheb protein levels were analyzed by WB. Protein ratios were calculated following Image J densitometric analysis, $\left({ }^{*} \mathrm{P}<\right.$ $0.05, * * \mathrm{P}<0.01)$.

of microRNA-155 mimics on autophagy in EA.hy926 endothelial cells by fluorescence microscopy. Consistent with the western blotting results, the protein accumulation of Beclin-1 was significantly up-regulated in cells transfected with microRNA-155 mimics (Fig. $2 \mathrm{~F}$ ). Thus, both results suggest that microRNA-155 induces autophagosome accumulation in EA.hy926 endothelial cells.

However, the autophagosome accumulation attributes to either enhanced autophagic activation or a block in the downstream degradation [29]. The autophagic flux should be analysed in EA.hy926 endothelial cells. First, we examined the protein level of P62/SQSTM1 by western blot, which is degraded during the late stage of autophagy, and negative correlates with autophagic activation [28]. As shown, up-regulation of microRNA-155 resulted in reduction of P62/SQSTM1 protein levels (Fig. 2E and $\mathrm{I},{ }^{*} \mathrm{P}<0.05$, ${ }^{*} \mathrm{P}<0.01$ ). In addition, cells were treated with bafilomycin $\alpha 1$ (BAF) which could prevent autophagolysosome formation [30]. BAF stimulation caused significant up-regulation of LC3-II and P62/SQSTM1 protein levels in microRNA-155 mimics-transfected cells (Fig. $2 \mathrm{E}, \mathrm{H}$ and $\mathrm{I}, * \mathrm{P}<0.05,{ }^{* *} \mathrm{P}<0.01$ ). 


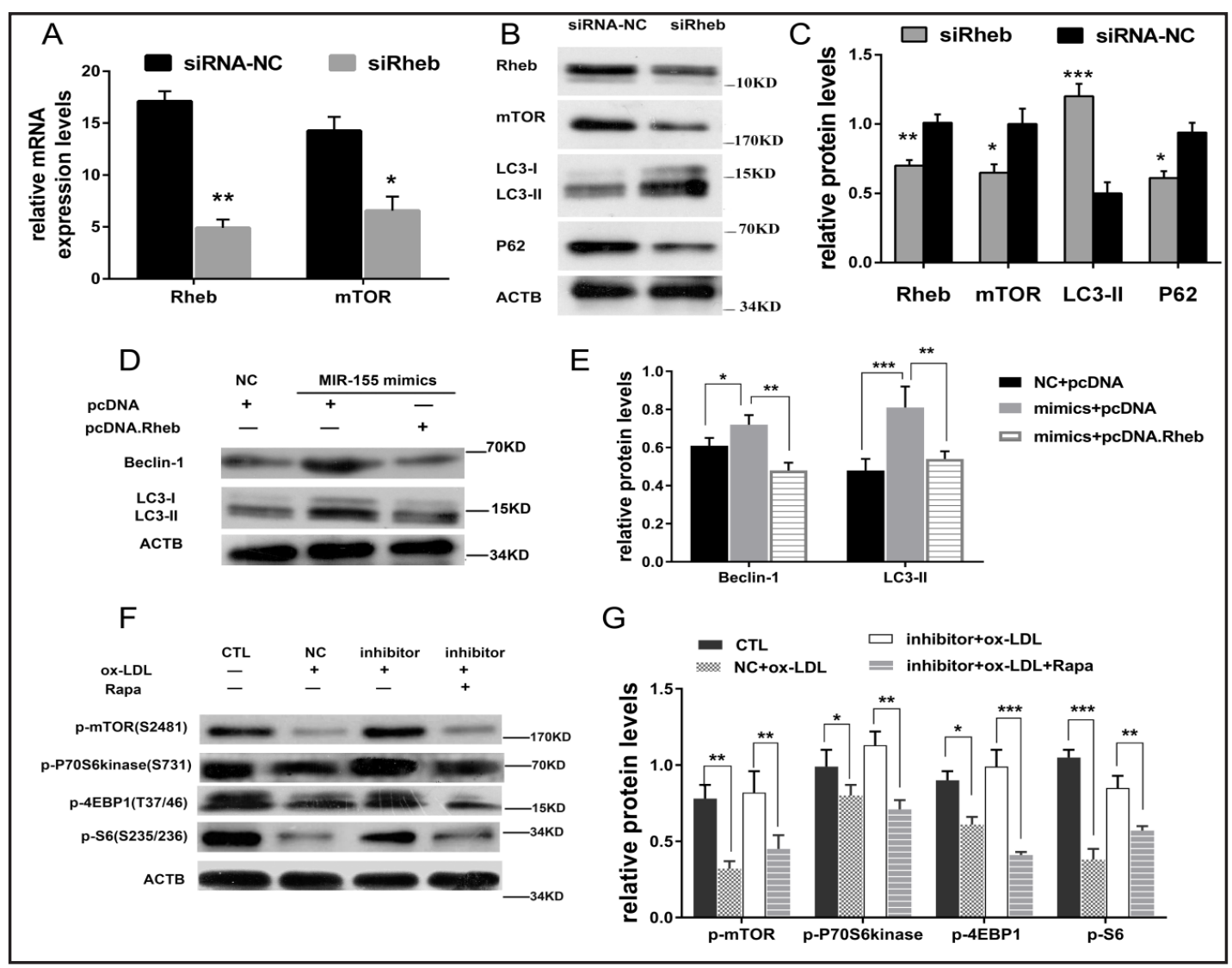

Fig. 5. MiR-155 induces autophagy via suppression of Rheb/mTOR pathway. (A) The mRNA expression of Rheb and mTOR were decreased in siRheb transfected cells, $\left({ }^{*} \mathrm{P}<0.05,{ }^{*} \mathrm{P}<0.01\right)$. (B-C) Western blot analysis of Rheb, mTOR, LC3-I/II, P62 in cells transfected with siRheb or siRNA-NC, $\left(* \mathrm{P}<0.05,{ }^{* *} \mathrm{P}<0.01\right.$, $* * * \mathrm{P}<0.001)$. (D-E) Western blot analysis of LC3-I/II, Beclin-1 in cells co-transfected with miR-155 and pcDNA-Rheb/empty pcDNA plasmid, $\left({ }^{*} \mathrm{P}<0.05,{ }^{* *} \mathrm{P}<0.01,{ }^{* * *} \mathrm{P}<0.001\right)$. (F-G) EA.hy926 cells were activated by ox-LDL, then p-mTOR, p-P70S6kinase, p-4EBP1 and p-S6 protein levels were detected by Western blot after Rapa and/or miR-155 inhibitor treatment, ( $\left.{ }^{*} \mathrm{P}<0.05,{ }^{* *} \mathrm{P}<0.01,{ }^{* * *} \mathrm{P}<0.001\right)$. Protein ratios were calculated following Image J densitometric analysis.

Therefore, these results indicates that microRNA-155 promotes both accumulation of autophagosome and autophagic degradation, suggesting the autophagic activation in EA.hy926 endothelial cells.

Inhibition of MicroRNA-155 suppresses ox-LDL-induced autophagy in endothelial cells

To further explore the physiological relevance of microRNA-155 in EA.hy926 endothelial cells, we inhibited endogenous microRNA-155 expression under ox-LDL stress $(80 \mathrm{ug} / \mathrm{ml})$, then repeated LC3 and P62/SQSTM1 protein assays by western blot. As shown, ox-LDL increased LC3-II and decreased P62/SQSTM1 levels, reflecting an enhancement of autophagic activation. However, inhibition of microRNA-155 significantly reduced P62/SQSTM1 degradation and LC3-II levels in cells treated with ox-LDL (Fig. 3A-B, ${ }^{* *} \mathrm{P}<0.01,{ }^{* * *} \mathrm{P}<0.001$ ). Hence, these data demonstrates that inhibition of microRNA-155 could suppress ox-LDLinduced autophagic activation.

MicroRNA-155 directly interacts with 3'-UTR of Rheb

Ras homolog enriched in brain (Rheb) has been reported to be a direct target of microRNA-155 [23]. To understand the biological role that underlies the interaction KARGER 


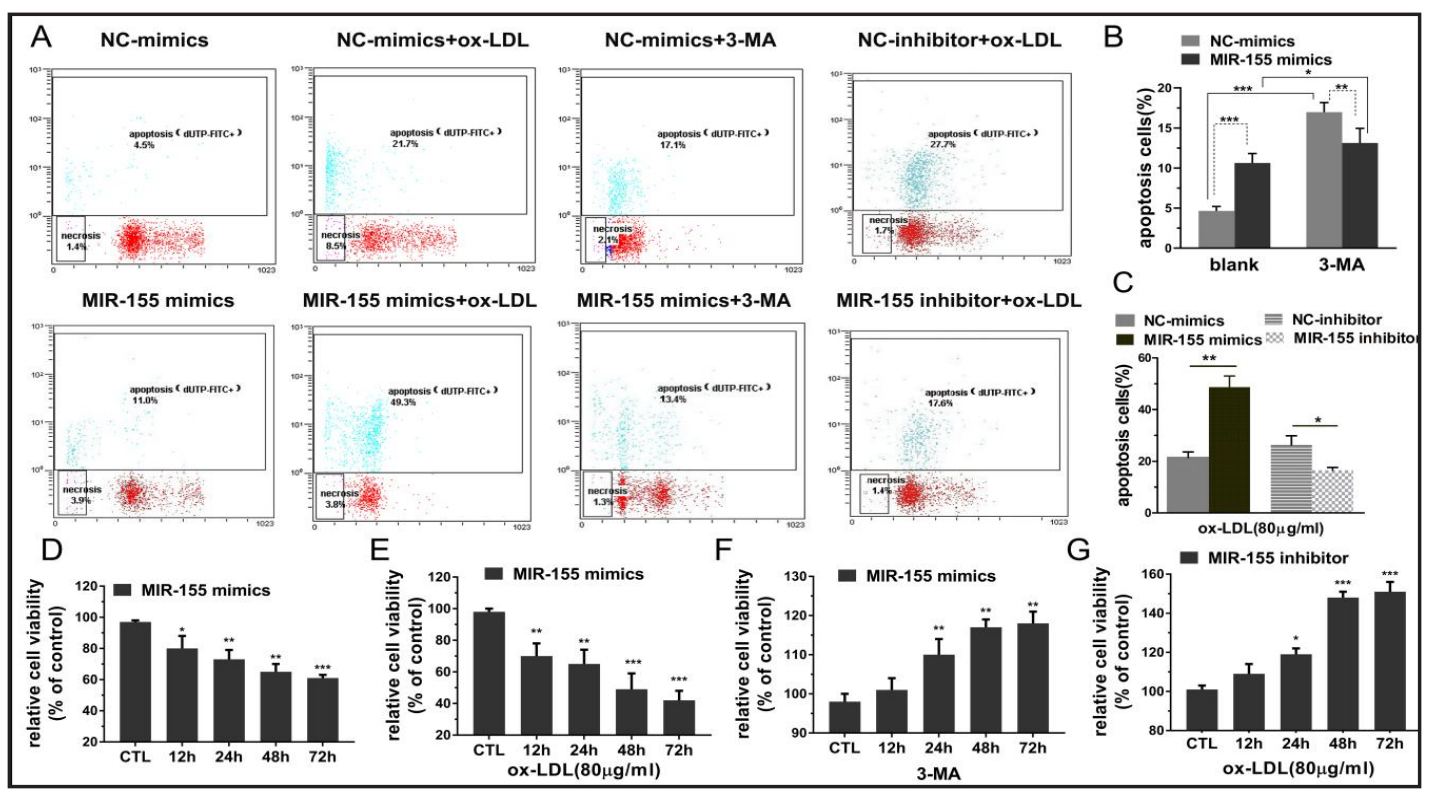

Fig. 6. MiR-155 attenuates cell viability and induces apoptosis under ox-LDL stress. (A-C) Cell apoptosis was measured by flow cytometry at $24 \mathrm{~h}$ after transfection, $\left({ }^{*} \mathrm{P}<0.05,{ }^{* *} \mathrm{P}<0.01,{ }^{* * *} \mathrm{P}<0.001\right)$. (D-E) MiR155 over -expression attenuated cell viability, $\left({ }^{*} \mathrm{P}<0.05,{ }^{* *} \mathrm{P}<0.01,{ }^{* * *} \mathrm{P}<0.001\right)$. (F) MiR-155 enhanced cell viability under 3 -MA stress, $\left({ }^{* *} \mathrm{P}<0.01\right)$. (G) MiR-155 inhibition improved cell viability under ox-LDL stress, $\left({ }^{*} \mathrm{P}<0.05,{ }^{* * *} \mathrm{P}<0.001\right)$.

between microRNA-155 and Rheb in endothelial cells, we cloned a construct with a fragment of the 3'-UTR of Rheb mRNA with the presumptive microRNA-155 binding sequence into a firefly dual-luciferase reporter vector for co-transfection with vehicle control into EA.hy926 endothelial cells. Luciferase reporter assay was performed at $24 \mathrm{~h}$ after transfection with wild-type (WT) or mutated (MUT) plasmids. In cells co-transfected with 3'-UTR-WT and microRNA-155, the activity of luciferase was changed significantly. (Fig. $4 \mathrm{~A}-\mathrm{B},{ }^{*} \mathrm{P}<0.05$ ). For further detection, we transfected microRNA-155 mimic or inhibitor into endothelial cells. As shown, up-regulation of microRNA-155 led to a significant decrease, while inhibition of it led to an obvious increase of Rheb protein levels, relative to the control group (Fig. 4C-D, ${ }^{*} \mathrm{P}<0.05,{ }^{* *} \mathrm{P}<0.01$ ). Thus, microRNA-155 could directly target Rheb.

\section{MicroRNA-155 induces autophagy via suppression of Rheb/mTOR pathway}

We considered whether knocking down of Rheb expression could phenocopy the effect of microRNA-155 on autophagy. We transfected small interfering RNA (siRNA)Rheb or siRNA-NC into EA.hy926 endothelial cells. By qRT-PCR and western blot assays, we validated that expression of Rheb in siRheb group was lower than in siRNA-NC group (Fig. 5A-C, ${ }^{* *} \mathrm{P}<0.01$ ). Similar to microRNA-155, depletion of endogenous Rheb induced a significant increase of LC3-II and a reduction of P62 protein levels (Fig. $5 \mathrm{~B}-\mathrm{C},{ }^{*} \mathrm{P}<0.05$, $* * * \mathrm{P}<0.001)$. Then, we co-transfected microRNA-155 mimic and plasmid into endothelial cells. The pcDNA-Rheb plasmid was designed to carry the open reading frame of human Rheb without 3'-UTR. The empty pcDNA was a control. As shown, LC3-II and Beclin-1 levels were lowered in cells co-transfected with microRNA-155 mimics and pcDNA-Rheb plasmid (Fig. 5D-E, ${ }^{*} \mathrm{P}<0.05,{ }^{* *} \mathrm{P}<0.01,{ }^{* * *} \mathrm{P}<0.001$ ). The results suggest that depletion of endogenous Rheb induces autophagy, whereas over-expression of Rheb could overcome microRNA-155 induced autophagy in endothelial cells.

Furthermore, we found that depletion of endogenous Rheb also attenuated the expression of mammalian target of rapamycin (mTOR) at both transcription and translation levels, which is known as a central negative regulator of autophagy (Fig. $5 \mathrm{~A}-\mathrm{C},{ }^{*} \mathrm{P}<0.05$ ). We 
transfected cells with microRNA-155 inhibitor or inhibitor- NC under ox-LDL stress, then checked the protein levels of phospho-mTOR (S2481) and its downstream targets phosphoP70S6kinase (S731), phospho-4EBP1 (T37/46) and phospho-S6 (S235/236) by western blot. We showed that ox-LDL induced down-regulation of p-mTOR, p-P70S6 kinase, p-4EBP1 and p-S6 proteins. But, the amounts of these proteins were increased while microRNA-155 was inhibited. More intriguing results, when we used rapamycin to attenuate mTOR activity in microRNA-155 inhibited cells, the p-P70S6 kinase, p-4EBP1, p-S6 levels are significantly decreased again (Fig. 5F-G, ${ }^{* *} \mathrm{P}<0.01,{ }^{* * *} \mathrm{P}<0.001$ ). In the light of the above results, we indicate that ox-LDL-induced microRNA-155 targets the Rheb/mTOR signaling pathway, and enhances the autophagic activation.

\section{MicroRNA-155 attenuates cell viability and induces apoptosis under ox-LDL stress}

As autophagy is implicated in cellular survival to a large extent, we assumed that microRNA- 155 might also protect cells from injury. Contrary to our expectation, we observed that microRNA-155 attenuated cell viability and significantly increased the average apoptotic cell fractions, especially under ox-LDL $(80 \mathrm{ug} / \mathrm{ml})$ stress (Fig. $6 \mathrm{~A}-\mathrm{E} * \mathrm{P}<0.05$, $\left.{ }^{* *} \mathrm{P}<0.01,{ }^{* * *} \mathrm{P}<0.001\right)$. Whereas, inhibition of microRNA-155 improved cell viability and retarded apoptosis (Fig.6A, $\mathrm{C}$ and $\mathrm{G}{ }^{*} \mathrm{P}<0.05,{ }^{* *} \mathrm{P}<0.01,{ }^{* * *} \mathrm{P}<0.001$ ). Furthermore, cells were pretreated with a classical autophagy inhibitor-3-MA [31] (5nM) for $4 \mathrm{~h}$, then transfected with microRNA- 155 mimics or NC-mimics. Interestingly, inhibition of autophagy increased the apoptotic cell fractions in both microRNA-155 and NC-mimics transfected cells, but microRNA-155 could reduce the apoptotic effect caused by 3 -MA and improve cell viability (Fig. 6A, B and $\mathrm{F}^{*} \mathrm{P}<0.05,{ }^{* *} \mathrm{P}<0.01,{ }^{* * *} \mathrm{P}<0.001$ ). These collective results demonstrate that microRNA-155 plays different roles in diverse circumstances, and might act as a possible link-bridge between autophagy and apoptosis.

\section{Discussion}

Accumulating evidence indicates that endothelial dysfunction drives initiation, development and even rupture of atherosclerotic plaques [32]. Therefore, it is necessary to focus on the potential targets of endothelial dysfunction, which in turn would provide novel prevention and treatment strategies [33].

In recent years, microRNAs have been of great concern in atherosclerosis $[13,34]$. It is imperative to illustrate that microRNA-155 impacts and regulates development of atherogenesis. Besides, previous findings coincide with our results which identified that microRNA-155 is significantly up-regulated following exposure of cells to ox-LDL, and correlates with atherosclerosis $[35,36]$. Muller et al. observed that ox-LDL intensifies autophagy in endothelial cells compared with cells treated with native LDL or negative control group [37]. So, we hypothesized that microRNA-155 was involved in the process of endothelial autophagy. Our results showed that ectopic high expression of microRNA-155 increases both formation and degradation of autolysosome in EA.hy926 endothelial cells, and vice versa when inhibited microRNA-155. It is implied that ox-LDL-induced microRNA-155 acts as a potent activator of autophagy in endothelial cells.

We then predicted and confirmed Rheb, a small G protein, as a target of microRNA-155 in oxLDL-induced endothelial cells. Previous studies have demonstrated that over-expression of Rheb in mammalian cells directly leads to mTOR activation [38]. As an upstream regulator of mTOR, Rheb is regulated by kinases, such as Akt, AMP-activated protein kinase (AMPK), and glycogen synthase kinase-3, which control Rheb through modulating the heterodimer consisting of tuberous sclerosis 1/tuberous sclerosis 2 complex (TSC1/TSC2) [39]. The mTOR signaling integrates and transmits signals to regulate cell growth, metabolism, ribosomal biogenesis, as well as autophagy $[40,41]$. Zhai et al. indicated that selective inhibition of $\mathrm{PI} 3 \mathrm{~K} / \mathrm{Akt} / \mathrm{mTOR}$ signaling pathway regulates autophagy of macrophages and vulnerability of atherosclerotic plaques [42]. Peng et al. reported that mTOR activation inhibites oxLDL- 
induced autophagy and apoptosis in vascular endothelial cells and restricted atherosclerosis in apolipoprotein E-/-mice [43]. We noticed that knockdown of Rheb could attenuate the expression of mTOR, which has been characterized as a negative regulator of autophagy machinery, and thus activate autophagy in endothelial cells. Furthermore, we demonstrated that ox-LDL inhibits mTOR phosphorylation via microRNA-155 up-regulation, and the inhibitory effect on p- mTOR is associated with down-regulation of p-P70S6 kinase, p-4EBP and the substrate of p-P70S6 kinase, p- S6. Thus, microRNA-155 plays an inductive role by suppressing Rheb-mediated mTOR/P70S6kinase/ 4EBP signaling pathway in endothelial autophagy.

Attention must be paid on the effects of autophagy on cellular homeostasis. It is predominantly recognized as a pro-survival pathway during cellular stress in plaque formation [44]. For instance, apoptosis of statin-induced smooth muscle cells (SMCs) could be partially rescued by autophagy inducer [45]. Likewise, the protective role of verapamil stereoisomers in promoting neointima formation is directly associated with the onset of autophagy following vascular injury [46]. On the contrary, several lines of evidence indicated that autophagy plays a harmful role in atherosclerosis. Inhibition of autophagy stimulates nitric oxide production and protects endothelial function [47]. Autophagic death of SMCs results in plaque destabilization owing to the reduced synthesis of collagen and thinning of the fibrous cap [48]. In Ischemia/Reperfusion injury, iNOS induces vascular endothelial cell migration and apoptosis via autophagy [49]. Recently, microRNAs have been focused on the crosstalk between apoptosis and autophagy by the action of some common proteins, such as Beclin-1, p53 and DAPK [50-53]. Here we discovered that microRNA-155 induces not only autophagy but also apoptosis in endothelial cells, while inhibition of it alleviates ox-LDLinduced apoptosis and consecutively improves cell viability rate. Whereas, microRNA-155 reduces the apoptotic effect caused by 3-MA and enhances cell viability. Though, statistically significant does not necessarily mean biologically relevant, the results could still suggest that microRNA-155 has diverse roles in varied circumstances, and might act as a possible link-bridge between autophagy and apoptosis.

Autophagic activity represents a prospective future therapeutic target in atherosclerotic diseases [54]. However, despite the discovery of many specific genes and the dissection of signaling pathways involved in the regulation of autophagy provided therapeutic approaches which are highly limited. With the above findings microRNA-155 would be one of the potential targets through co-intervention of autophagy and apoptosis in endothelial cells. Indeed, the existed achievement is far from enough, and exploring the effect of microRNA-155 on autophagy in vivo would be necessary in our future research. In spite of all the valuable knowledge, additional studies on microRNA-155 are needed to be clarified in the pathological mechanism of atherosclerosis.

\section{Acknowledgements}

We would like to thank all members of the Cardiology Department of Kunming General Hospital and the Medical Research Center of The Third Affiliated Hospital of Kunming Medical University for assistance, we thank Doctor Xin Liu for equipment support.

\section{Disclosure Statement}

The authors have not disclosed any potential conflicts of interest.

\section{References}

1 Mathers CD, Loncar D: Projections of global mortality and burden of disease from 2002 to 2030. PLoS Med 2006;3:e442. 


\section{Cellular Physiology Cell Physiol Biochem 2017;43:1436-1448

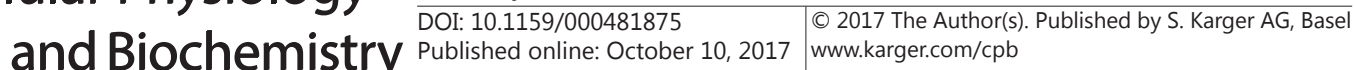

2 Libby P: Molecular and cellular mechanisms of the thrombotic complications of atherosclerosis. J Lipid Res 2009;50:S352-357.

3 Cahill PA, Redmond EM: Vascular endothelium-Gatekeeper of vessel health. Atherosclerosis 2016;248: 97109.

-4 Aburto MR, Hurlé JM, Varela-Nieto I, Magariños M: Autophagy during vertebrate development. Cells 2012;1:428-448.

5 Levine B, Kroemer G: Autophagy in the pathogenesis of disease. Cell 2008;132:27-42.

6 Chao CL, Chuang CP, Cheng YF, Lee KR, Chang Y, Cheng SP, Chan WK, Ho FM: The Protective Role of Autophagy in Matrix Metalloproteinase-Mediated Cell Transmigration and Cell Death in High-GlucoseTreated Endothelial Cells. Inflammation 2016;39:830-838.

7 Kroemer G, Levine B: Autophagic cell death: the story of a misnomer. Nat Rev Mol Cell Biol 2008;9:10041010.

8 Chao J, Wang X, Zhang Y, Zhu T, Zhang W, Zhou Z, Yang J, Han B, Cheng Y, Tu X, Yao H: Role of MCPIP1 in the Endothelial-Mesenchymal Transition Induced by Silica. Cell Physiol Biochem 2016;40:309-325.

-9 Mei Y, Thompson MD, Cohen RA, Tong X: Autophagy and oxidative stress in cardiovascular diseases. Biochim Biophys Acta 2015;1852:243-251.

10 Martinet W, Schrijvers DM, De Meyer GR: Molecular and cellular mechanisms of macrophage survival in atherosclerosis. Basic Res Cardiol 2012;107:297.

11 Goodall ML, Fitzwalter BE, Zahedi S, Wu M, Rodriguez D, Mulcahy-Levy JM, Green DR, Morgan M, Cramer SD, Thorburn A: The Autophagy Machinery Controls Cell Death Switching between Apoptosis and Necroptosis. Dev Cell 2016;37:337-349.

12 Winter J, Jung S, Keller S, Gregory RI, Diederichs S: Many roads to maturity: microRNA biogenesis pathways and their regulation. Nat Cell Biol 2009;11:228-234.

-13 Zhang T, Tian F, Wang J, Jing J, Zhou SS, Chen YD: Endothelial Cell Autophagy in Atherosclerosis is Regulated by miR-30-Mediated Translational Control of ATG6. Cell Physiol Biochem 2015;37:1369-1378.

14 Zhou Z, You Z: Mesenchymal Stem Cells Alleviate LPS-Induced Acute Lung Injury in Mice by MiR-142a5p-Controlled Pulmonary Endothelial Cell Autophagy. Cell Physiol Biochem 2016;38:258-266.

15 Du F, Yu F, Wang Y, Hui Y, Carnevale K, Fu M, Lu H, Fan D: MicroRNA-155 deficiency results in decreased macrophage inflammation and attenuated atherogenesis in apolipoprotein E-deficient mice. Arterioscler Thromb Vasc Biol 2014;34:759-767.

16 Zhang J, Zhao F, Yu X, Lu X, Zheng G: MicroRNA-155 modulates the proliferation of vascular smooth muscle cells by targeting endothelial nitric oxide synthase. Int J Mol Med 2015;35:1708-1714.

17 Tian FJ, An LN, Wang GK, Zhu JQ, Li Q Zhang YY, Zeng A, Zou J, Zhu RF, Han XS, Shen N, Yang HT, Zhao XX, Huang S, Qin YW, Jing Q: Elevated microRNA-155 promotes foam cell formation by targeting HBP1 in atherogenesis. Cardiovasc Res 2014;103:100-110.

18 Nazari-Jahantigh M, Wei Y, Noels H, Akhtar S, Zhou Z, Koenen RR, Heyll K, Gremse F, Kiessling F, Grommes J, Weber C, Schober A: MicroRNA-155 promotes atherosclerosis by repressing Bcl6 in macrophages. J Clin Invest 2012;122:4190-4202.

19 Yang Y, Yang L, Liang X, Zhu G: MicroRNA-155 Promotes Atherosclerosis Inflammation via Targeting SOCS1. Cell Physiol Biochem 2015;36:1371-1381.

20 Li X, Kong D, Chen H, Liu S, Hu H, Wu T, Wang J, Chen W, Ning Y, Li Y, Lu Z: miR-155 acts as an antiinflammatory factor in atherosclerosis-associated foam cell formation by repressing calcium-regulated heat stable protein 1. Sci Rep 2016;6:21789.

21 Wu XY, Fan WD, Fang R, Wu GF: Regulation of microRNA-155 in endothelial inflammation by targeting nuclear factor (NF)-кB P65. J Cell Biochem 2014;115:1928-1936.

22 Zhu H, Wu H, Liu X, Li B, Chen Y, Ren X, Liu CG, Yang JM: Regulation of autophagy by a beclin 1-targeted microRNA, miR-30a, in cancer cells. Autophagy 2009;5:816-823.

23 Wang J, Yang K, Zhou L, Minhaowu, Wu Y, Zhu M, Lai X, Chen T, Feng L, Li M, Huang C, Zhong Q Huang X: MicroRNA-155 promotes autophagy to eliminate intracellular mycobacteria by targeting Rheb. PLoS Pathog 2013;9:e1003697.

24 Chen L, Jiang K, Jiang H, Wei P: miR-155 mediates drug resistance in osteosarcoma cells via inducing autophagy. Exp Ther Med 2014;8:527-532. 


\section{Cellular Physiology Cell Physiol Biochem 2017;43:1436-1448

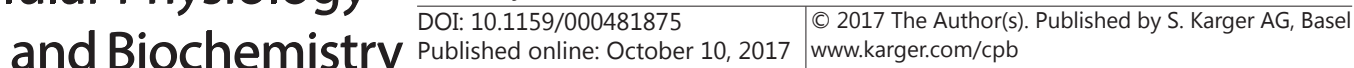

Lv et al.: MicroRNA-155-Rheb and Endothelial Autophagy

-25 Krzeszinski JY, Wei W, Huynh H, Jin Z, Wang X, Chang TC, Xie XJ, He L, Mangala LS, Lopez-Berestein G, Sood AK, Mendell JT, Wan Y: miR-34a blocks osteoporosis and bone metastasis by inhibiting osteoclastogenesis and Tgif2. Nature 2014;512:431-435.

-26 Janssen HL, Reesink HW, Lawitz EJ, Zeuzem S, Rodriguez-Torres M, Patel K, van der Meer AJ, Patick AK, Chen A, Zhou Y, Persson R, King BD, Kauppinen S, Levin AA, Hodges MR: Treatment of HCV infection by targeting microRNA. N Engl J Med 2013;368:1685-1694.

27 Buttari B, Profumo E, Businaro R, Saso L, Capoano R, Salvati B, Riganò R: Oxidized haemoglobin-driven endothelial dysfunction and immune cell activation: novel therapeutic targets for atherosclerosis. Curr Med Chem 2013;20:4806-4814.

-28 Mizushima N, Yoshimori T, Levine B: Methods in mammalian autophagy research. Cell 2010;140:313- 326.

-29 Zhang XJ, Chen S, Huang KX, Le WD: Why should autophagic flux be assessed. Acta Pharmacol Sin 2013;34:595-599.

-30 Kobayashi S, Koujin T, Kojidani T, Osakada H, Mori C, Hiraoka Y, Haraguchi T: BAF is a cytosolic DNA sensor that leads to exogenous DNA avoiding autophagy. Proc Natl Acad Sci U S A 2015;112:7027-7032.

-31 Wu Y, Wang X, Guo H, Zhang B, Zhang XB, Shi ZJ, Yu L: Synthesis and screening of 3-MA derivatives for autophagy inhibitors. Autophagy 2013;9:595-603.

32 Grassi D, Desideri G, Ferri C: Cardiovascular risk and endothelial dysfunction: the preferential route for atherosclerosis. Curr Pharm Biotechnol 2011;12:1343-1353.

-33 Jensen HA, Mehta JL: Endothelial cell dysfunction as a novel therapeutic target in atherosclerosis. Expert Rev Cardiovasc Ther 2016;14:1021-1033.

34 Miano JM, Long X: The short and long of noncoding sequences in the control of vascular cell phenotypes. Cell Mol Life Sci 2015;72:3457-3488.

-35 Yan H, Wang S, Li Z, Zhao W, Wang Z, Sun Z, Pan Y, Zhu J: Upregulation of miRNA-155 expression by OxLDL in dendritic cells involves JAK1/2 kinase and transcription factors YY1 and MYB. Int J Mol Med 2016;37:1371-1378.

-36 Ma YL, Ma ZJ, Wang M, Liao MY, Yao R, Liao YH: MicroRNA-155 induces differentiation of RAW264.7 cells into dendritic-like cells. Int J Clin Exp Pathol 2015;8:14050-14062.

-37 Muller C, Salvayre R, Nègre-Salvayre A, Vindis C: Oxidized LDLs trigger endoplasmic reticulum stress and autophagy: prevention by HDLs. Autophagy 2011;7:541-543.

-38 Martin TD, Chen XW, Kaplan RE, Saltiel AR, Walker CL, Reiner DJ, Der CJ: Ral and Rheb GTPase activating proteins integrate mTOR and GTPase signaling in aging, autophagy, and tumor cell invasion. Mol Cell 2014;53:209-220.

39 Heard JJ, Fong V, Bathaie SZ, Tamanoi F: Recent progress in the study of the Rheb family GTPases. Cell Signal 2014;26:1950-1957.

40 Dunlop EA, Tee AR: mTOR and autophagy: a dynamic relationship governed by nutrients and energy. Semin Cell Dev Biol 2014;36:121-129.

41 Jia G, Aroor AR, Martinez-Lemus LA, Sowers JR: Overnutrition, mTOR signaling, and cardiovascular diseases. Am J Physiol Regul Integr Comp Physiol 2014;307:R1198-1206.

-42 Zhai C, Cheng J, Mujahid H, Wang H, Kong J, Yin Y, Li J, Zhang Y, Ji X, Chen W: Selective inhibition of PI3K/ Akt/mTOR signaling pathway regulates autophagy of macrophage and vulnerability of atherosclerotic plaque. PLoS One 2014; 9:e90563.

43 Peng N, Meng N, Wang S, Zhao F, Zhao J, Su L, Zhang S, Zhang Y, Zhao B, Miao J: An activator of mTOR inhibits oxLDL-induced autophagy and apoptosis in vascular endothelial cells and restricts atherosclerosis in apolipoprotein $\mathrm{E}^{-} /^{-}$mice. Sci Rep 2014;4:5519.

44 Mizumura K, Choi AM, Ryter SW: Emerging role of selective autophagy in human diseases. Front Pharmacol 2014;5:244.

45 Martinet W, De Bie M, Schrijvers DM, De Meyer GR, Herman AG, Kockx MM: 7-ketocholesterol induces protein ubiquitination, myelin figure formation, and light chain 3 processing in vascular smooth muscle cells. Arterioscler Thromb Vasc Biol 2004;24:2296-2301.

46 Salabei JK, Balakumaran A, Frey JC, Boor PJ, Treinen-Moslen M, Conklin DJ: Verapamil stereoisomers induce antiproliferative effects in vascular smooth muscle cells via autophagy. Toxicol Appl Pharmacol 2012;262:265-272. 


\section{Cellular Physiology Cell Physiol Biochem 2017;43:1436-1448

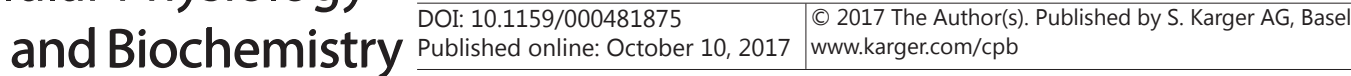 \\ Lv et al.: MicroRNA-155-Rheb and Endothelial Autophagy}

-47 Pestana CR, Oishi JC, Salistre-Araújo HS, Rodrigues GJ: Inhibition of autophagy by chloroquine stimulates nitric oxide production and protects endothelial function during serum deprivation. Cell Physiol Biochem 2015;37:1168-1177.

48 Levine B, Yuan J: Autophagy in cell death: an innocent convict. J Clin Invest 2005;115:2679-2688.

49 Zhu T, Yao Q, Wang W, Yao H, Chao J: iNOS Induces Vascular Endothelial Cell Migration and Apoptosis Via Autophagy in Ischemia/Reperfusion Injury. Cell Physiol Biochem 2016;38:1575-1588.

-50 Siddiqui MA, Mukherjee S, Manivannan P, Malathi K: RNase L Cleavage Products Promote Switch from Autophagy to Apoptosis by Caspase-Mediated Cleavage of Beclin-1. Int J Mol Sci 2015;16:17611-17636.

-51 Livesey KM, Kang R, Vernon P, Buchser W, Loughran P, Watkins SC, Zhang L, Manfredi JJ, Zeh HJ, Li L, Lotze MT, Tang D: p53/HMGB1 complexes regulate autophagy and apoptosis. Cancer Res 2012;72:1996-2005.

52 Gandesiri M, Chakilam S, Ivanovska J, Benderska N, Ocker M, Di FP, Feoktistova M, Gali-Muhtasib H, RaveFränk M, Prante O, Christiansen H, Leverkus M, Hartmann A, Schneider-Stock R: DAPK plays an important role in panobinostat-induced autophagy and commits cells to apoptosis under autophagy deficient conditions. Apoptosis 2012;17:1300-1315.

53 Su Z, Yang Z, Xu Y, Chen Y, Yu Q: MicroRNAs in apoptosis, autophagy and necroptosis. Oncotarget 2015;6:8474-8490.

54 Orogo AM, Gustafsson ÅB: Therapeutic targeting of autophagy: potential and concerns in treating cardiovascular disease. Circ Res 2015;116:489-503. 